\title{
ASYMPTOTIC EXPANSIONS OF GAUSSIAN INTEGRALS
}

\author{
BY RICHARD S. ELLIS ${ }^{1}$ AND JAY S. ROSEN ${ }^{2}$
}

Function space integrals are useful in many areas of mathematics and physics. Physical problems often give rise to function space integrals depending on a parameter and the asymptotics with respect to the parameter yield important information about the original problem. The purpose of this note is to describe the asymptotic expansions of a wide class of Gaussian function space integrals. Related work has been done by [Varadhan], [Schilder], [Pincus], [Donsker-Varadhan], and [Castro]. All asymptotic expansions previously obtained assume a nondegeneracy condition which assures that one never strays too far from the realm of Gaussian processes. Our results cover both the nondegenerate case and the degenerate case, the analysis of the latter being much more subtle. In the degenerate case, the leading asymptotic behavior is non-Gaussian.

Let $\boldsymbol{P}_{\boldsymbol{A}}$ be a mean zero Gaussian probability measure with covariance operator $\boldsymbol{A}$ on a separable Hilbert space $H$. Our methods can also handle certain Banach spaces, such as $C[0,1]$, which are important in applications. Let $\psi$ and $F$ be suitably bounded, real $C^{\infty}$ functionals on $H$. We study the asymptotics of

$$
J_{n}:=\int \psi(Y / \sqrt{n}) e^{-n F(Y / \sqrt{n})} d P_{A}(Y)
$$

as $n \rightarrow \infty$.

If $f$ and $g$ are real-valued functions on $\mathbf{R}$, then Laplace's method tells us that the asymptotics of $\int_{\mathrm{R}} f(x) \exp (-n g(x)) d x$ are determined by the behavior of $g$ near its minimum points [Erdélyi, §2.4]. Formally, $J_{n}$ in (1) can be written as

$$
\int \psi(Y / \sqrt{n}) e^{-n F(Y / \sqrt{n})-\left(A^{-1} Y, Y\right) / 2} d Y=\int \psi(Y / \sqrt{n}) e^{-n G(Y / \sqrt{n})} d Y
$$

where $G(Y):=F(Y)+\left(A^{-1} Y, Y\right) / 2$. By analogy with the situation on $\mathbf{R}$, we expect the asymptotics of $J_{n}$ to be determined by the behavior of $G$ near its minimum points. The expressions in (2) are purely formal since the symbol $d Y$

Received by the editors November 30, 1979 and, in revised form, February 27, 1980.

1980 Mathematics Subject Classification. Primary 60B99, 60G15.

Key words and phrases. Asy mptotic expansion, Gaussian integral, nondegenerate minimum point, degenerate minimum point.

1 Alfred P. Sloan Research Fellow. Research supported in part by NSF Grant MPS 76-06644-A01.

2 Research supported in part by NSF Grant PHY 77-02172.

(c) 1980 American Mathematical Society $0002-9904 / 80 / 0000-0302 / \$ 02.25$ 
is supposed to represent the nonexistent translation invariant measure on $\mathrm{H}$. Also, $\left(A^{-1} Y, Y\right)$ is defined only for $Y \in D\left(A^{-1 / 2}\right)$, which in general is only a dense subset of $H$.

For simplicity, assume that $G$ has a unique minimum point $Y^{*}$. We necessarily have

$$
G^{\prime}\left(Y^{*}\right)=0, \quad G^{\prime \prime}\left(Y^{*}\right) \geqslant 0 \text { on } H .
$$

Here, $G^{\prime}\left(Y^{*}\right)$ and $G^{\prime \prime}\left(Y^{*}\right)$ are respectively the element of $H$ and the linear operator on $H$ defined by the first and second Fréchet derivatives of $G$ at $Y^{*}$. We say that $Y^{*}$ is nondegenerate if $K:=\operatorname{ker}\left(G^{\prime \prime}\left(Y^{*}\right)\right)=\{0\}$. In this case, we can write for $\|Y\|$ small

$$
G\left(Y^{*}+Y\right)=G\left(Y^{*}\right)+1 / 2\left(G^{\prime \prime}\left(Y^{*}\right) Y, Y\right)+\text { error term. }
$$

Thus, the important term in the exponent of (2) is $1 / 2\left(G^{\prime \prime}\left(Y^{*}\right) Y, Y\right)$, giving rise to a Gaussian measure with covariance operator $\left(G^{\prime \prime}\left(Y^{*}\right)\right)^{-1}$. The asymptotics of $J_{n}$ are derived by expanding around this measure. This is the intuition behind Theorem 1. In the special case of Wiener measure, the theorem was proved by [Schilder].

In Theorem 1 and in its analogue for the degenerate case, Theorem 2, we suppose that $\psi$ and $F$ are real $C^{\infty}$ functionals on $H$ and that there exist constants $b_{1}>0, b_{2} \geqslant 0,0 \leqslant b_{3}<1 /(2\|A\|), b_{4} \geqslant 0$ such that

$$
|\psi(Y)| \leqslant b_{1} e^{b_{2}\|Y\|^{2}}, \quad F(Y) \geqslant-b_{3}\|Y\|^{2}-b_{4}
$$

for $Y \in H$. These bounds are sufficient to assure that $J_{n}$ exists.

THEOREM 1. Suppose that $G$ has a unique minimum point $Y^{*}$ and that $Y^{*}$ is nondegenerate. Then $B:=\left(G^{\prime \prime}\left(Y^{*}\right)\right)^{-1}$ is the covariance operator of a mean zero Gaussian measure $P_{B}$ on $H$. Also, $\exp \left(n G\left(Y^{*}\right)\right) J_{n}$ has the asymptotic expansion

$$
\epsilon^{n G\left(Y^{*}\right)} J_{n}=\sum_{j=0}^{M} n^{-j} \Gamma_{j}+O\left(n^{-(M+1)}\right) \quad \text { as } n \rightarrow \infty,
$$

where $M \geqslant 0$ is any integer and the $\left\{\Gamma_{j}\right\}$ are functionals. The sum in (6) is obtained by expanding

$$
\begin{aligned}
{\left[\operatorname{det}\left(I+A F^{\prime \prime}\left(Y^{*}\right)\right)\right]^{-1 / 2} \psi\left(Y^{*}+Y / n^{1 / 2}\right) } & \\
\cdot \exp \left(-n\left[F\left(Y^{*}+Y / n^{1 / 2}\right)-\right.\right. & F\left(Y^{*}\right)-\left(F^{\prime}\left(Y^{*}\right), Y / n^{1 / 2}\right) \\
& \left.\left.-1 / 2\left(F^{\prime \prime}\left(Y^{*}\right) Y / n^{1 / 2}, Y / n^{1 / 2}\right)\right]\right)
\end{aligned}
$$

in powers of $n^{-1 / 2}$ through order $n^{-M}$ and then by integrating term by term with respect to $d P_{B}$ over $H$. The leading term in (6) equals

$$
\left[\operatorname{det}\left(I+A F^{\prime \prime}\left(Y^{*}\right)\right)\right]^{-1 / 2} \psi\left(Y^{*}\right) \text {. }
$$


REMARK. In (6), we must multiply $J_{n}$ by $\exp \left(n G\left(Y^{*}\right)\right)$ in order to cancel the contribution of $G\left(Y^{*}\right)$ when (4) is substituted into (2). This also applies to the degenerate case (see (13)). Only even powers of $n^{-1 / 2}$ appear in (6) since $P_{B}$ is mean zero.

We now discuss the degenerate case. When $K:=\operatorname{ker}\left(G^{\prime \prime}\left(Y^{*}\right)\right)$ is nonempty, the above analysis completely breaks down and we can no longer expand around a Gaussian measure. Let us assume for simplicity that $\operatorname{dim}(K)=1$. In such a case, we say that $Y^{*}$ is simply degenerate. Let $U \in K$ be a unit vector and let $\tau$ denote the orthogonal projection onto $K^{\perp}$. We write $Y \in H$ in the form

$$
Y=z U+X \in K \oplus K^{\perp}
$$

where $z:=(Y, U)$ and $X:=\tau Y$. Following (4), we examine $G\left(Y^{*}+Y\right)$ for $\|Y\|$ small. If we use the coordinates $z, X$ from (8), we find for some $m>2$

$$
\begin{aligned}
G\left(Y^{*}+Y\right)=G\left(Y^{*}\right) & +\frac{1}{2}\left(G^{\prime \prime}\left(Y^{*}\right) X, X\right)+\frac{z^{m}}{m !} G^{(m)}\left(Y^{*} ; U^{m}\right) \\
& +(\text { cross terms in } z, X)+(\text { error terms in } z, X) .
\end{aligned}
$$

Unfortunately, when inserted into (2), the cross terms in (9) contribute to the leading asymptotics of $J_{n}$. We introduce new local coordinates which allow us to keep track of these contributions in an efficient way.

The operator $\tau G^{\prime \prime}\left(Y^{*}\right) \tau$ is invertible on $K^{\perp}$. By the implicit function theorem, the relation

$$
\Phi=-\left(\tau G^{\prime \prime}\left(Y^{*}\right) \tau\right)^{-1} \tau\left[F^{\prime}\left(Y^{*}+Y+\Phi\right)-F^{\prime}\left(Y^{*}\right)-F^{\prime \prime}\left(Y^{*}\right)(Y+\Phi)\right]
$$

defines a unique $C^{\infty}$ function $\Phi=\Phi(Y) \in K^{\perp}$ for $\|Y\|$ sufficiently small. Now suppose that for small $z$ we can write

$$
G\left(Y^{*}+z U+\Phi(z U)\right)=G\left(Y^{*}\right)+\lambda z^{k}+O\left(z^{k+1}\right),
$$

where $\lambda \neq 0$ and $k<\infty$ is an integer. Since $Y^{*}$ is a simply degenerate minimum point, we must have $\lambda>0, k$ even, and one can show $k \geqslant 4$. With this notation, writing $Y=z U+\Phi(z U)+X, X \in K^{\perp}$, we find

$$
\begin{aligned}
G\left(Y^{*}+Y\right) & =G\left(Y^{*}+z U+\Phi(z U)+X\right) \\
& \left.=G\left(Y^{*}\right)+\lambda z^{k}+1 / 2\left(G^{\prime \prime}\left(Y^{*}\right) X, X\right)+\text { (error terms in } z, X\right) .
\end{aligned}
$$

This is the correct analogue of (4) in the simply degenerate case. This approximate decoupling of $G$ along the directions $K$ and $K^{\perp}$ is the basic intuition behind Theorem 2. We emphasize that $\Phi(z U)$ can be computed to any order in $z$ by a straightforward iteration and that $\lambda$ and $k$ are also readily determined.

TheOREM 2. Suppose that $G$ has a unique minimum point $Y^{*}$ and that $Y^{*}$ is simply degenerate. Then $\tau G^{\prime \prime}\left(Y^{*}\right) \tau$ is invertible on $K^{\perp}$ with inverse operator denoted by $B_{\perp} . B_{\perp}$ is the covariance operator of a mean zero Gaussian measure $P_{B_{\perp}}$ concentrated on $K^{\perp}$. Suppose that (11) holds. Then $\exp \left(n G\left(Y^{*}\right)\right) J_{n}$ 
has the asymptotic expansion

$$
e^{n G\left(Y^{*}\right)} J_{n}=n^{1 / 2-1 / k}\left\{\sum_{j=0}^{M} n^{-j / k} \Gamma_{j, k}+O\left(n^{-(M+1) / k}\right)\right\} \quad \text { as } n \rightarrow \infty,
$$

where $M \geqslant 0$ is any integer and the $\left\{\Gamma_{j, k}\right\}$ are functionals. Let $W_{n}(z):=z U / n^{1 / k}$ $+\Phi\left(z U / n^{1 / k}\right)$. The sum in (13) is obtained by expanding

$$
\begin{aligned}
& \frac{1}{[2 \pi(A U, U) D]^{1 / 2}} \psi\left(Y^{*}+W_{n}(z)+X / n^{1 / 2}\right) \\
& \cdot \exp \left(-n\left[G\left(Y^{*}+W_{n}(z)\right)-G\left(Y^{*}\right)-\lambda\left(\frac{z}{n^{1 / k}}\right)^{k}\right]\right) \\
& \cdot \exp \left(-n\left[F\left(Y^{*}+W_{n}(z)+X / n^{1 / 2}\right)-F\left(Y^{*}+W_{n}(z)\right)\right.\right. \\
& \left.\left.-\left(F^{\prime}\left(Y^{*}+W_{n}(z)\right), X / n^{1 / 2}\right)-1 / 2\left(F^{\prime \prime}\left(Y^{*}\right) X / n^{1 / 2}, X / n^{1 / 2}\right)\right]\right)
\end{aligned}
$$

in powers of $n^{-1 / k}$ through order $n^{-M / k}$ and then by integrating term by term with respect to $\exp \left(-\lambda z^{k}\right) d z d P_{B_{\perp}}(x)$ over $\mathbf{R} \times K^{\perp}$. In (14),

$$
D:=\operatorname{det}\left[I+\tau \rho A \rho \tau F^{\prime \prime}\left(Y^{*}\right) \tau\right] \text {, }
$$

where $\rho$ is the bounded operator on $H$ defined by

$$
\rho Y:=Y-[(A U, Y) /(A U, U)] U
$$

The leading term in (13) equals

$$
n^{1 / 2-1 / k}(2 \pi(A U, U) D)^{-1 / 2}\left(\int_{-\infty}^{\infty} e^{-\lambda z^{k}} d z\right) \psi\left(Y^{*}\right)
$$

We see that in the simply degenerate case, the asymptotic expansion is obtained by expanding around a non-Gaussian measure in the degenerate direction $K$ and around a Gaussian measure in the nondegenerate directions $K^{\perp}$.

We sketch the proof of Theorem 2. By [Donsker-Varadhan, Theorem 6.2], [Varadhan, §3], for any $\delta>0$ there exists $c=c(\delta)>0$ such that

$$
e^{n G\left(Y^{*}\right)} \int_{\left\|n^{-1 / 2} Y-Y^{*}\right\| \geqslant \delta} e^{-n F(Y / \sqrt{n})} d P_{A}(Y)=O\left(e^{-n c}\right) \quad \text { as } n \rightarrow \infty .
$$

On the set $\left\|n^{-1 / 2} Y-Y^{*}\right\|<\delta$, we argue formally. Provided $\delta$ is sufficiently small, we may change variables on this set from $Y$ to $n^{1 / 2}\left(Y^{*}+W_{n}(z)+X / n^{1 / 2}\right)$, where $W_{n}(z)=z U / n^{1 / k}+\Phi\left(z U / n^{1 / k}\right)$. Write $S_{n}$ for the set $\left\{z, X: \| W_{n}(z)+\right.$ $\left.X / n^{1 / 2} \|<\delta\right\}$ and $\psi_{n}$ for the expression involving $\psi$ in (14). Using (12) with explicit forms for the error terms, we see that formally 


$$
\begin{aligned}
& e^{n G\left(Y^{*}\right)} J_{n}=e^{n G\left(Y^{*}\right)} \int_{\left\|n^{-1 / 2} Y-Y^{*}\right\|<\delta} \psi(Y / \sqrt{n}) e^{-n G(Y / \sqrt{n})} d Y+O\left(e^{-n c}\right) \\
& =n^{1 / 2-1 / k} \int_{S_{n}} \psi_{n} \exp \left(-n\left[G\left(Y^{*}+W_{n}(z)+X / n^{1 / 2}\right)-G\left(Y^{*}\right)\right]\right) d z d X+O\left(e^{-n c}\right) \\
& =n^{1 / 2-1 / k} \int_{S_{n}} \psi_{n} \zeta_{n} e^{-\lambda z^{k}} d z d P_{B_{\perp}}(X)+O\left(e^{-n c}\right) \text { as } n \rightarrow \infty
\end{aligned}
$$

where $\zeta_{n}$ denotes the product of the exponentials in (14). We expand $\psi_{n} \zeta_{n}$ in powers of $n^{-1 / k}$ through order $n^{-M / k}$. Using the bound $P_{B_{\perp}}(\|X\| \geqslant \alpha) \leqslant$ $C_{1} \exp \left(-C_{2} \alpha^{2}\right)$ for $\alpha>0\left(C_{1}, C_{2}\right.$ positive constants), we control the errors and show that (16) gives rise to the same expansion (13) as does (14). This formal argument gives the right answer except for the factor $[2 \pi(A U, U) D]^{-1 / 2}$ in (14). The operator $\tau \rho A \rho \tau$, which appears in the definition of $D$, inverts $\tau A^{-1} \tau$ on $K^{\perp}$.

Theorems 1 and 2 assume that $G$ has a unique minimum point which is either nondegenerate or simply degenerate. Our methods also handle the case where $G$ has finitely many minimum points, including the case of degenerate minimum points which are not simply degenerate. Function space integrals with nonisolated degenerate minimum points play an important role in recent physics; see, for example, [Coleman]. We will treat this case in a forthcoming paper. We have also derived limit theorems for probability measures related to $J_{n}$. These will appear elsewhere. These limit theorems and the asymptotic expansions discussed in this note were inspired by a model in statistical mechanics [Ellis-Rosen, (1), (2)].

\section{BIBLIOG RAPHY}

1. P. E. Castro, Asymptotic formulas for expectations with respect to a diffusion on a small time interval, Ph.D. dissertation, New York University, 1975.

2. S. Coleman, The uses of instantons, Lectures delivered at the 1977 International School of Subnuclear Physics, Ettore Majorane.

3. M. D. Donsker and S. R. S. Varadhan, Asymptotic evaluation of certain Markov process expectations for large time. III, Comm. Pure Appl. Math. 29 (1976), 389-461.

4. R. S. Ellis and J. S. Rosen, Asymptotics of certain random fields on a circle, Proceedings of Colloquium on Random Fields: Rigorous Results in Statistical Mechanics and Quantum Field Theory, Esztergom, Hungary, June 1979. Seria Colloquia Mathematica Societatis Jānos Bolyai, North-Holland (to appear).

5. Laplace's method for Gaussian integrals with an application to statistical mechanics (to appear).

6. A. Erdélyi, Asymptotic expansions, Dover, New York, 1956.

7. M. Pincus, Gaussian processes and Hammerstein integral equations, Trans. Amer. Math. Soc. 134 (1968), 193-216.

8. M. Schilder, Some asymptotic formulas for Wiener integrals, Trans. Amer. Math Soc. 125 (1966), 63-85.

9. S. R. S. Varadhan, Asymptotic probabilities and differential equations, Comm. Pure Appl. Math. 19 (1966), 261-286.

DEPARTMENT OF MATHEMATICS AND STATISTICS, UNIVERSITY OF MASSACHUSETTS, AMHERST, MASSACHUSETTS 01003 